\title{
Paradojas de las profesiones de la información en la sociedad del conocimiento
}

\author{
Miriam Vieira da Cunha \\ Helena Maria Tarchi Crivellari \\ Universidade Federal de Santa Catarina (Brasil)
}

Las profesiones de la información viven, en este momento, un desafío y una transformación de una profundidad sin precedentes desde la invención de la imprenta en el siglo XV... Pero, a pesar de los cambios traídos por la historia, la misión fundamental de las profesiones de la información — servir a la sociedad respondiendo a sus necesidades de información — seguirá una necesidad estable y permanente. (Starr, 1996)

\section{Resumen}

A partir de un análisis de los conceptos de ocupación y de profesión, se discuten las transformaciones que ocurren en el sistema de las profesiones, en el mundo del trabajo y de la formación profesional. Estas discusiones tienen como fondo el ambiente de la sociedad del conocimiento y los cambios económicos y organizacionales, bajo la óptica de la sociología de las profesiones. Se abordan especialmente las profesiones que se ocupan del trato con la información. Se discuten las funciones de los profesionales de la información, su importancia y su posición en el mundo del trabajo, sus interrelaciones con profesionales de otras disciplinas en nuevos ambientes informacionales y los conflictos que son consecuencia de estas interacciones. Se enfatiza la importancia de las funciones ligadas al ciclo de la información, destacando la necesidad de que el profesional de la información se muestre más activo, más presente y más visible en las discusiones ligadas a las políticas nacionales de información y en la construcción de un proyecto de sociedad de la información. Se lanzan ideas sobre el futuro del profesionalismo, de los monopolios y de las relaciones profesionales en un ambiente de cambios crecientes.

Palabras clave: Sociedad del conocimiento. Profesionales de la información. Mercado de trabajo.

\begin{abstract}
After an analysis of professional and occupational concepts, the transformations that have taken place over the last ten years in the profession, the work environment and professional qualifications are discussed. As a background, the study has the society of knowledge as well as economic, social and organizational changes and is treated from the perspective of professional
\end{abstract}


sociology, dealing in particular with professions connected with information. The study discusses the functions of information professionals, their importance and position in the work environment, their inter-relations with professionals of other areas of knowledge in new informational environments and the conflicts brought about by these interactions. It emphasizes the breadth and the importance of tasks related to the informational cycle, highlighting the necessity for information professionals to have a more active, current and visible role in discussions on national information policies and the construction of a project for the information society. It suggests ideas for the future of professionalism, monopolies and professional relations in an increasingly changing environment.

Key words: Knowledge society. Information professionals. Labour market.

\section{Introducción}

Las profesiones, de acuerdo con Freidson (1998), "son los agentes que crean y hacen avanzar el conocimiento en los asuntos humanos y del Estado". En este sentido, y a partir de un análisis de los conceptos de ocupación y de profesión, este trabajo pretende discutir las transformaciones que están siendo implantadas, en los últimos diez años, en el mundo del trabajo y de la formación profesional. Estas discusiones tienen como marco el ambiente de la sociedad del conocimiento y los cambios económicos, sociales y organizacionales, y se da bajo la ótica de la sociología de las profesiones, abordando especialmente las profesiones ligadas al trato con la información. El objetivo es analizar los impactos de los cambios que la globalización ha impuesto a la organización, a los ambientes profesionales y al mundo del trabajo en general.

Los términos profesión y ocupación se refieren a actividades especializadas, condicionadas al tipo de estratificación social y al grado de división del trabajo predominante en una determinada sociedad. Esencialmente, lo que distingue los dos términos es, precisamente, la existencia de un cuerpo de saberes científicos. La profesión, desde el punto de vista de la sociología de las profesiones, posee esa dimensión cognitiva ligada a saberes específicos apenas accesibles para el grupo profesional que los posee. En ese sentido, la formación profesional asume un papel crucial, porque el título constituye el principal fundamento del derecho a la autoridad. La profesión posee, todavía, una dimensión normativa y valorativa que define su papel social y jerárquico en el conjunto de la sociedad y en relación con otras profesiones. Son las asociaciones profesionales, los sindicatos y el Estado los que desempeñan ese papel normativo. La noción de ocupación, en Brasil, desde el punto de vista del Ministerio del Trabajo y del Empleo, es ya la unidad del sistema de clasificación de ocupaciones (Brasil, 2002). En este contexto, es definida como el conjunto articulado de funciones, tareas y operaciones que constituyen las obligaciones atribuidas a un trabajador destinadas a la obtención de productos o servicios.

Scire. 12 : 2 (jul.-dic. 2006) 13-26. ISSN 1135-3716. 
Profesión y ocupación se constituyen en construcciones históricas resultantes de las relaciones sociales de producción predominantes en cada época y lugar (Crivellari, 2000).

Según Bourdieu (1989), una institución pública encargada de producir estadísticas y clasificaciones (como la Clasificación Brasileña de Ocupaciones) puede ser analizada como un instituto del Estado que, al producir taxonomías oficiales investidas de un valor casi jurídico, sobre todo en las relaciones entre patrones y empleados, tiende a fijar las jerarquías y, al hacerlo, consagra una relación de fuerza entre los agentes respecto de los nombres de las profesiones y de las ocupaciones (1), componente esencial de la identidad social. La gestión de los nombres es una gestión de raridad material de un grupo profesional y es, por lo tanto, objeto de luchas. La entrada en una profesión está casi siempre subordinada a la posesión de un título universitario. "El título profesional o escolar es una especie de regla jurídica de percepción social, un ser percibido que es garantido como un derecho. Es un capital simbólico [2] institucionalizado, legal y apenas legítimo" (Bordieu, 1989, p. 148).

La estructura cognitiva de las profesiones debe ser legitimada por la sociedad. En este sentido, cada profesión es relacionada con una comunidad a través de un contrato implícito, el cual da el derecho exclusivo del ejercicio profesional a cambio de la seguridad, la calidad y la eficacia de los servicios ofrecidos. Estos derechos derivan del conocimiento que legitima su campo de trabajo (Curry y Wergin, 1993). Pueden, asimismo, incluir el monopolio de la práctica profesional, el control de la formación y del reclutamiento de sus miembros. Según Haug (1973), el monopolio del conocimiento especializado que una profesión posee asegura su lugar en la jerarquía del sistema profesional, su estatus y su prestigio. Moore (1970) precisa además que, cuanto más sistematizados están los conocimientos de un campo de actividad, más se garantiza el monopolio de su espacio. Por lo tanto, para este autor, el control del conocimiento se establece a partir de las relaciones que existen entre la práctica profesional y valores como legitimidad cultural, racionalidad y eficacia. Más aún, añade que el conocimiento común es indispensable para mantener la unión de un grupo profesional.

Según Abbott (1988), el conjunto de las profesiones forma un sistema en el cual las profesiones separan espacios más o menos legitimados de acuerdo con el poder que ejerce cada una de ellas. El autor define este sistema como una estructura que relaciona las profesiones entre ellas de tal forma que el movimiento de una afecta a las otras. La evolución de este sistema de interdependencias depende de los ajustes que se realizan entre las profesiones, ajustes que son consecuencia de la forma en que los diversos grupos profesionales controlan sus conocimientos y competencias. En este sentido, el control de la profesión viene determinado por el dominio de las abstracciones que generan la práctica profesional. Los límites de cada 
jurisdicción profesional están siempre en disputa. Es la historia de estas disputas la que determina la historia de las profesiones. Así, la habilidad de las profesiones para mantener su jurisdicción o su espacio resulta, en parte, del prestigio de su sistema de conocimiento. En este sentido, cuanto mayor sea el poder de abstracción teórica de una profesión, más sólida será esta en el espacio social y en el sistema profesional (Cunha, 2000).

La evolución de las profesiones es, de esta forma, el resultado de sus interrelaciones, las cuales vienen determinadas por la manera en que los grupos profesionales controlan sus conocimientos y habilidades. Existen, según Abbott (1988), dos formas de realizar este control. La primera enfatiza la técnica por sí misma, en las ocupaciones o métiers. El grupo que controla la ocupación controla su técnica. La segunda es el control del conocimiento. Aquí, las habilidades técnicas evolucionan hacia un sistema de conocimiento abstracto y el control de la profesión está fundamentado en el control de las abstracciones que generan esta práctica. Las prácticas pueden, en este sentido, delegarse en otros trabajadores. Para este autor, esta abstracción es la característica que mejor identifica las profesiones.

En su estudio sobre la evolución de las profesiones a través de sus interrelaciones, Abbott (1988) afirma que cada una de ellas ocupa un lugar específico en el sistema y desarrolla sus actividades en varios tipos de jurisdicción. Ese lugar viene determinado a través de un núcleo central, un campo de actividad bastante delimitado, sobre el cual el grupo ejerce un control completo y legal. A través de este control, según especifica el autor, cada profesión intentará excluir a otros trabajadores de su campo.

La fuerza y el éxito de una profesión están, de esta manera, legitimados por la delimitación clara de su campo de competencia, la delimitación de un espacio propio de acción, y a través de su interacción con otras profesiones.

Cronin, Stiffler y Day (1983) dividen el espacio ocupado por cada profesión en heartland, hinterland y horizon. El heartland puede ser considerado como el núcleo central de la profesión. Según Abbott, cada profesión lucha para mantener este núcleo, sobre el cual tiene un control legal y completo. El hinterland es definido por Cronin como la periferia de la misma, y el horizon como sus márgenes. Para Abbott y para Cronin, Stiffler y Day, los nuevos espacios profesionales surgen a partir del hinterland o periferia, donde muchas veces hay confluencias entre profesiones próximas. En este espacio, los profesionales son más valorizados por su expertise que por su título. De ahí van a surgir las nuevas luchas de poder profesional, de influencias y de legitimidad para formar nuevas jurisdicciones, según el sentido que Abbott da a este término. Cronin (1988a, p. 279) compara las profesiones con "camisas de fuerza" (3) que pretenden poner en orden una masa disforme o, dicho de otra forma, son un intento de imponer un carácter unitario a una población variada. A partir del cambio de base disciplinar y de la admisión de nue-

Scire. 12 : 2 (jul.-dic. 2006) 13-26. ISSN 1135-3716. 
vos insights y abordajes, cada profesión es forzada a "adoptar" aquellos que ejercen actividades marginales o incluso a excluirlos de su campo. Este movimiento es parte de la "lucha por la supervivencia de los espacios profesionales" enfatizada por Abbott.

El fenómeno central de la vida profesional es el vínculo entre la profesión y su trabajo - lo que Abbott denomina jurisdicción-. En este sentido, analizar el desarrollo profesional es analizar la forma en que este vínculo es visto por la estructura social.

La habilidad de una profesión para garantizar su jurisdicción está fundamentada en el poder y el prestigio de su conocimiento académico, el cual legitima el trabajo profesional, aclarando sus orígenes y relacionándolo con valores culturales.

Cada profesión viene determinada por una serie de tareas cuya fuerza o debilidad se establecen a través del trabajo profesional. Como ninguna de estas tareas es permanente, las profesiones construyen un sistema de interacciones - una ecología- Las diferentes profesiones compiten entre sí dentro de este sistema. De vez en cuando las tareas cambian, son absorbidas o transformadas por fuerzas externas como la tecnología o los sistemas políticos, y llevan al sistema a ajustarse a estos cambios.

Existe el reconocimiento de una especialidad cuando un grupo profesional dispone de un savoir-faire que no es compartido con otros grupos, de modo que se confiere a cada uno de sus miembros un lugar específico en la sociedad. En este caso, el grupo tendrá el control total de un determinado espacio - como ocurre, por ejemplo, con la medicina- . Algunas veces este control está subordinado a la intervención de otro grupo profesional —es el caso de las profesiones paramédicas-. Cuando los límites de una profesión no están claramente definidos, profesionales de otras áreas "invaden" su campo, lo que provoca una disputa por el espacio. La evolución de las profesiones fundamenta, por consiguiente, estas relaciones, así como la competición que existe entre ellas.

De esta forma, es el control de un espacio y del campo de actividad profesional el que crea los conflictos y la interdependencia entre las profesiones. Analizar su desarrollo es, en este sentido, analizar los vínculos existentes entre cada profesión y su campo de trabajo. Cada profesión defiende intereses que le son propios. Muchas veces, sin embargo, las características de una ocupación no son exclusivas de un grupo, sino que derivan de especialidades de otras actividades. Además, cuando la demanda hecha a un grupo profesional por el mercado de trabajo sobrepasa su capacidad de respuesta, este grupo pierde su espacio o se subdivide. Es lo que ocurrió con las especializaciones ligadas a la ingeniería, por ejemplo. La aparición de una nueva ocupación significa, de esta forma, que esta tiene actividades específicas suficientemente valoradas y que se diferencian de otras, con una utilidad 
reconocida públicamente. Así, las especializaciones profesionales surgen cuando existe una diferenciación en la estructura que forma las profesiones: tipo de clientes, local de trabajo, etcétera.

Delorme y André (1983), al discutir la relación entre cualificación, empleo y formación, la definen, en la misma línea de razonamiento que Abbott, como un espacio de lucha. Entendiendo la cualificación como la capacidad de efectuar una tarea determinada, se van a encontrar, de un lado, los patrones, que intentan adaptar al máximo las cualificaciones a sus empleos, y, del otro, los trabajadores, que buscan alcanzar el máximo de cualificación con el fin obtener autonomía respecto a los empleos. El resultado final de esta lucha va a depender del juego de fuerzas, lo que rompe con la idea de un determinismo estricto entre las estructuras productivas y las de cualificación. A las escuelas les toca el dilema de lanzar al mercado de trabajo títulos universitarios que jamás están completamente adaptados a la estructura laboral. Las políticas de innovación constantes en el seno de las organizaciones agravan este problema, ya que las innovaciones tecnológicas alteran la calidad y el tipo de profesionales que la escuela debe aportar a los sistemas de trabajo. Hay que tener en cuenta que la rapidez de los cambios que tienen lugar en las empresas no se corresponde con el tiempo necesario para la readaptación del sistema de formación a las cualificaciones requeridas en este ambiente.

Delorme y André (1983) enfatizan, asimismo, las aspiraciones individuales. En este sentido, en el plano de las aspiraciones personales, la escuela favorece un sistema de orientación fuertemente liberal, no autoritario, en el cual la promesa de éxito social descansa sobre la posesión de un título universitario. Esa promesa no siempre se cumple, debido al fuerte desequilibrio entre el número de titulados lanzados al mercado y la cantidad y la naturaleza de las plazas de trabajo disponibles. Hay, por consiguiente, un juego de fuerzas entre los profesionales, sus consejos, sindicatos y asociaciones, las escuelas y el mundo del trabajo.

\section{Reflexiones sobre los profesionales de la información}

El saber científico, garantizado por el título universitario, y la protección del título profesional, ejercida por los pares a través de asociaciones, son condiciones fundamentales para el ejercicio oficial y el reconocimiento social de cada profesión. Se puede decir que el bibliotecario se encuentra en esa situación. El profesional de la información es una resultante que precisa ser analizada más detenidamente, lo que se propone hacer aquí.

Además de la escuela y de las asociaciones de clase, como quedó claro en el apartado anterior, hay una tercera condición que define una profesión: se trata del modo de ejercerla o de sus características en términos de organización de sus procesos de trabajo. Un ejemplo clásico lo encontramos en las llamadas profesiones liberales, ejercidas individualmente por el profesional en oficina propia, sin una re- 
lación patronal, tal como ocurre frecuentemente con los abogados y los médicos, por ejemplo.

En el caso de los bibliotecarios esa situación no es habitual. Normalmente, las actividades específicas de la biblioteconomía son ejercidas en el interior de bibliotecas u otras unidades de información (centros de información y documentación, principalmente) con profesionales contratados para ese fin, provistos de título y fiscalizados, en el caso brasileño por el consejo profesional. El trabajo en las unidades de información es una actividad casi siempre pública, pues a ellas tienen acceso los ciudadanos en general, incluso si están ubicadas en el interior de instituciones privadas, como las escuelas privadas. Las bibliotecas, habitualmente, no tienen fines lucrativos. Por esta razón, difícilmente se podría calcular el mayor valor del trabajo desempeñado en estas instituciones. Así, se podría sugerir que el bibliotecario se encontraría tradicionalmente en una situación semejante a la de los cadres, tal como los define Boltanski (1982). O sea, un profesional dentro de una organización.

Esa situación pasaría a ser cuestionada a partir de los años ochenta teniendo en cuenta el uso ampliado y generalizado de las TIC, que acarrearía innovaciones en el ejercicio de la biblioteconomía a la vez que abriría nuevas alternativas de trabajo en este ámbito. Este panorama merece atención especial, ya que es en ese contexto donde van a surgir los profesionales de la información.

El sector de la información es, por su propia naturaleza, un sector heterogéneo. La diversidad de los ambientes profesionales donde se realizan estas actividades, así como la de sus actores, y la interpenetración relativa de sus funciones hacen realmente difícil la caracterización de los espacios de información. En este sentido, los límites que separan las ocupaciones de este sector no están claros. En realidad, la evolución de la sociedad de la información da la impresión de una confusión de pistas, una yuxtaposición de espacios, de actores y de funciones.

La dificultad existente para delimitar este campo es también consecuencia de la incertidumbre de los papeles que estos profesionales deben desempeñar en una realidad donde los cambios tecnológicos son muy rápidos. De acuerdo con Sergean (1977, p. 7):

la dificultad para definir el campo de la información es consecuencia de la propia naturaleza de la información. Este recurso difiere de los otros porque no es el campo exclusivo de un grupo profesional, pero concierne a todos los individuos. Por esta razón, las cuestiones relativas a los límites de este campo pueden ser definidas solamente a través de reglas arbitrarias. Lo que le corresponde al especialista de la información en una determinada situación puede no corresponderle en otra [...] el trabajo informal es un trabajo que todo el mundo realiza, en el sentido en que, en determinadas situaciones, un abogado, un economista o un médico ejercen actividades de información.

Scire. 12 : 2 (jul.-dic. 2006) 13-26. ISSN 1135-3716. 
Según Cronin (1988b, p. 328), no existe una profesión de la información, sino un grupo grande y heterogéneo de profesionales que pueden ser calificados como pertenecientes a ese ámbito. Las funciones que ejercen y sus habilidades son tan diversas que es difícil colocarlas bajo un mismo "paraguas". En este sentido, la denominación profesional de la información es una rúbrica vaga, conveniente, que presupone un conjunto de categorías profesionales e ignora las diferencias de orientación, de formación básica y de las actividades ejercidas.

Aunque la noción de profesional de la información estuvo, hasta hace poco tiempo, ligada a grupos tradicionales con actividades bien definidas, como los bibliotecarios, los documentalistas, los museólogos y los archiveros (4), el desarrollo de estas profesiones "clásicas" del sector no impidió que profesionales procedentes de otros campos de actividad bastante diversos ocuparan este espacio, originando lo que Abbott denomina invasión de un campo profesional por otros grupos. De hecho, la ocupación de este espacio se ha intensificado durante los últimos diez años en el mercado de trabajo informativo.

Recientemente, esta diversidad se comprueba por la creación en países como Francia, Inglaterra, Estados Unidos, Dinamarca y Canadá, entre otros, de nuevos cursos y nuevas especialidades, tales como gestión de la información, gestión de recursos informativos, sistemas de información o gestión de sistemas de información. En Brasil, esta diversificación de cursos del área se ha hecho realidad en los últimos diez años. Hay que mencionar además la diversificación de espacios de actuación en esta área profesional. Además de los espacios "clásicos", como universidades, escuelas e instituciones públicas, donde el profesional ejerce su trabajo en unidades de información como bibliotecas, centros de documentación e información y archivos, existen actualmente unidades de información en cinematecas, filmotecas, videotecas, librerías y editoriales, sin olvidar Internet, que se ha revelado como un espacio cada vez más prometedor para estos profesionales, quienes también pueden trabajar en la gestión de archivos electrónicos o "en papel", oficinas de abogados, clínicas médicas y empresas diversas del sector industrial o de servicios, a través de lo que algunos llaman gestión del conocimiento.

Si tenemos en cuenta, además, que los programas de posgrado en ciencia de la información atraen — como docentes o discentes— a profesionales con formación básica en diferentes áreas del conocimiento, se podría decir que existe una efectiva "invasión" de otras áreas en las tradicionales profesiones de la información. Valga señalar la gran frecuencia, entre los profesionales anteriormente citados, de oriundi de la comunicación, ciencia de la computación, administración, psicología, economía, sociología, medicina o ingeniería, entre otras. En este sentido, se puede afirmar que existen dos movimientos paralelos en la actuación del profesional de la información: $a$ ) un movimiento de apertura del campo a profesionales 
de otras áreas del conocimiento; $b$ ) un movimiento de colonización de espacios diversificados de actuación profesional.

El término profesional de la información cubre un campo de actividades bastante extenso con denominaciones extremamente variadas, más o menos específicas de acuerdo con los actores que participan de estas actividades. Empezó a ser empleado en los Estados Unidos en la década de los ochenta para designar a

todo bibliotecario, documentalista, científico de la información, intermediario, etc., cuyo trabajo

- se fundamenta en la teoría y en la práctica de la creación, acceso, validación, organización, transmisión, investigación y difusión de la información;

- concierne a la gestión de los recursos de la información;

- utiliza tecnologías específicas de la ciencia de la información o de la gestión de la información. (Clausen, ápud Christensen, 1992, p. 68)

De acuerdo con Debons (ápud Chen, Raskin y Tebbets, 1984, pp. 166-167), la utilización creciente de la expresión profesional de la información denota una necesidad de convergencia de profesiones diferenciadas. A pesar de que su connotación continúa siendo amplia, este término sugiere, para este autor, una cierta unidad dentro de la diversidad. Si bien los profesionales de la información ejercen su actividad en diferentes tipos de instituciones y con clientelas diversificadas, en realidad es posible reagruparlos a partir de un conjunto de ideas que les son comunes y están asociadas a conceptos ligados a los flujos de información, como entrada, tratamiento, salida y memorización, entre otras.

En Brasil, la adopción de la nueva nomenclatura de la Clasificación Brasileña de Ocupaciones (CBO), que será más discutida en el próximo apartado, viene al encuentro de esta tendencia mundial al emplear el término profesionales de la información para nombrar, genéricamente, bibliotecarios, documentalistas y analistas de información (no obstante, los archiveros han sido excluidos de este grupo).

En los últimos diez años, esta expresión se está utilizando abundantemente y en Brasil se está empleando incluso en la literatura del área, la mayoría de las veces usando profesional de la información como sinónimo de bibliotecario. Este equívoco de terminología es consecuencia, a nuestro entender: $a$ ) de la desmaterialización y la creciente diversificación de los soportes informativos; $b$ ) del cambio de foco de competencia de las organizaciones; $c$ ) de una nueva diversificación de locales y de prácticas; $e$ ) de un aumento y una diversificación de alianzas entre profesionales de varios campos del conocimiento.

Uno de los problemas evidentes de este campo de actividad es que se desarrolló de tal manera que perdió su jurisdicción preliminar, lo que demuestra que hay una disputa por una nueva jurisdicción. De acuerdo con Abbott, este campo informativo no está ocupado completamente por ningún grupo profesional, pero sigue siendo un terreno extremadamente permeable que sigue caminos bastante diversificados. 
Por definición, una profesión debería, de acuerdo con Davies (1990), desarrollarse continuamente, a medida que surge un nuevo conocimiento, se desarrollan nuevos contenidos y aparecen nuevas demandas. Una profesión que no sigue este desarrollo está mal preparada para responder a las exigencias tecnológicas y sociales, de modo que su lugar es tomado inevitablemente por profesionales mejor preparados para desempeñar estos papeles.

\section{El caso brasileño: elementos para la reflexión}

En Brasil, el campo de actuación del profesional de la información recibió una definición oficial. Desarrollada en el ámbito del Ministerio del Trabajo y del Empleo, la Clasificación Brasileña de Ocupaciones (CBO) de 2002 ha introducido cambios muy significativos para los profesionales del área. Se trata del desmembramiento de las tres ocupaciones aglutinadas en la clasificación anterior, CBO1994, que reunía bibliotecarios, archiveros y museólogos (5). La nueva CBO no está más organizada a partir de las ocupaciones individualizadas, sino por la noción de familia ocupacional, que agrega funciones consideradas semejantes. En este caso específico tuvo lugar un desmembramiento en relación con la situación anterior. Mientras museólogos y archiveros continuaron integrando una misma familia ocupacional, los bibliotecarios pasaron a constituirse en la ocupación principal de una nueva familia creada para albergar los cambios en curso. De esta forma, bajo el nombre de profesionales de la información se encuentran hoy bibliotecarios, documentalistas y analistas de la información, dado que para el ejercicio de esas tres ocupaciones se exige la formación universitaria en biblioteconomía, según queda estipulado por la CBO. La nueva división refleja los cambios de estos espacios profesionales, pero no resuelve bien la relación de los bibliotecarios con los museólogos y archiveros - antiguos compañeros de profesión-, ya que la gestión de documentos abarca tanto bibliotecas como archivos y museos, porque son instituciones asemejadas en muchos aspectos, principalmente en la custodia de objetos físicos $\mathrm{y}$, frecuentemente, raros.

Así, según los criterios de la CBO, queda claro que el verdadero profesional de la información es, en Brasil, el propio bibliotecario, el cual es, de los tres componentes de la familia ocupacional profesionales de la información, el que está cualificado para ejercer la mayoría absoluta de las actividades típicas de aquella familia. Sin embargo, el término bibliotecario fue siendo subsumido dentro de profesional de la información.

Estas vacilaciones se reflejan, también, en el ámbito del sistema de formación profesional de estas categorías. Las escuelas acompañan los cambios del mundo laboral, pero estos no tienen un impacto inmediato sobre los planes curriculares, lo que, dicho sea de paso, es saludable, puesto que el llamado mercado de trabajo es fugaz. La actividad educacional responde a las innovaciones técnicas con re-

Scire. 12 : 2 (jul.-dic. 2006) 13-26. ISSN 1135-3716. 
lativa lentitud. Los tiempos en la escuela y en el sistema productivo no son iguales. Efectivamente, se abren diferentes procesos de trabajo para los portadores de los distintos títulos emitidos en este campo de formación. Sin embargo, la diversificación temática que se desdobla en el mundo de la información afecta de un modo diferente a la graduación y al posgrado. En la primera, la formación sigue siendo distinta en cada una de las subáreas tradicionales, archivología, museología y biblioteconomía. Además, se han creado nuevos cursos con títulos que incluyen la palabra información, tales como ciencia de la información, sistemas de información y gestión de la información, entre otros. Ya en el posgrado, en Brasil cada uno de los programas de maestría y doctorado en Ciencia de la Información intenta abarcar los diferentes temas y preocupaciones del área a través de sus líneas de investigación (Crivellari, 2003).

Los cambios en la estructura de la enseñaza evidencian el proceso de transformación del conjunto teórico y científico de la profesión, que busca para sus titulados la ampliación del cuadro de cualificaciones y de las posibilidades en el mercado de trabajo. En ese movimiento, las escuelas abandonan el nombre de biblioteconomía y pasan a utilizar el término ciencia de la información (6).

Observando el caso brasileño, desde el punto de vista del ejercicio profesional es posible verificar que este acompaña el movimiento de los países desarrollados, en términos de diversificación de las posibilidades de trabajo abiertas por las tecnologías de la información y la comunicación. No obstante, no tuvo lugar, como se imaginaba, una migración significativa de los puestos de trabajo tradicionales hacia sectores más dinámicos de la economía —empresas globalizadas y/o punto.com, por ejemplo- - Investigaciones recientes muestran, por el contrario, la permanencia del bibliotecario - nuestro principal profesional de la información, según la $\mathrm{CBO}$ - en empleos tradicionales, principalmente en bibliotecas públicas.

Cunha y Pereira (2003), investigando los egresos de los estudios de Biblioteconomía en Santa Catarina, constataron la existencia de una diversificación en la oferta de puestos de trabajo para los bibliotecarios, aunque actualmente la gran mayoría (cerca del 70\%) sigue trabajando en bibliotecas del Estado.

Resultados comparables a estos fueron analizados en una encuesta realizada en el estado del Espíritu Santo con los profesionales registrados en el Consejo Regional de Biblioteconomía (Rosemberg et ál., 2003). La encuesta de Jannuzzi (2003), que estudia los datos de la PNAD (Pesquisa Nacional por Amostra de Domicílios) sobre los profesionales de la información en Brasil, abarcando analistas de sistemas, estadísticos, economistas, periodistas, sociólogos, profesores universitarios y bibliotecarios/archiveros/museólogos (aún incluidos, como en la CBO-1994), constató que este último subgrupo presenta tasas superiores al $90 \%$ de empleados en el sector formal, en gran parte en el sector público y, predominantemente, en las áreas de educación y salud. Los datos muestran que el mercado de trabajo para el profesional de 
la información se mantiene en la biblioteca tradicional, con diversificación cualitativa, en otros sectores, pero no cuantitativa.

Para que conocer con detalle los cambios cualitativos que se produjeron en los procesos de trabajo del bibliotecario a partir de la introducción de las tecnologías de la información y la comunicación sería necesario desarrollar programas de investigación basados en el uso de métodos cualitativos en ciencias sociales, o en una combinación de estudios cualitativos y cuantitativos (SENAI, 1998). Entrevistas en profundidad a los profesionales identificados con las transformaciones en curso aportarían un material crucial para el entendimiento de esas modificaciones y, naturalmente, de quién es nuestro verdadero profesional de la información (graduación, posgrado, competencias, ámbito en que actúa, qué hace, etcétera).

Una vez más, surge la cuestión de la profesión del bibliotecario, ahora como profesión altamente institucionalizada, caracterizada por la existencia corporativa de un consejo profesional. Entonces, nos preguntamos: ¿por qué sus miembros permiten la ambigüedad oficial creada en torno al nombre de la profesión?, ¿qué valores simbólicos está adquiriendo al identificarse con las nuevas tecnologías?, ¿qué valores simbólicos está perdiendo al desvincular su nombre de los iconos tradicionales de la alta cultura: el libro, la biblioteca, el museo? (Bourdieu, 1989). Y, frente a los compromisos asumidos con la sociedad y sancionados por el Estado a través de los consejos profesionales para velar por estas tradicionales instituciones de la cultura, ¿qué se puede esperar?, ¿un título más valorado?, ¿más y mejores empleos? Poco probable, como lo ha demostrado largamente la historia reciente de la sociedad globalizada.

\section{Notas}

(1) En la edición citada, publicada en Lisboa, consta el término oficio, probablemente traduciendo el vocablo francés métier.

(2) Que no es posible adquirirlo con dinero.

(3) O usurpación, conforme a la visión de ciertos autores, como Magali Larson (sobre este punto, véase también Barbosa, 1993).

(4) Tal como indica el CNPq en su clasificación de las áreas científicas, que concibe la biblioteconomía, la archivística y la biblioteconomía en la misma subclase: "ciencias de la información".

(5) La CBO-1994 — cuya división completa llega hasta cinco dígitos—incluía en su subdivisión de tres dígitos a los bibliotecarios, archiveros y museólogos. Cada una de estas tres ocupaciones era observada aisladamente en la subdivisión de cinco dígitos. Sin prologar la discusión en este artículo, hemos de destacar, no obstante, que los cambios afectan a los análisis estadísticos de evolución del empleo para estas categorías ocupacionales en la medida en que impiden la comparación con las series históricas anteriores a la nueva CBO-2002.

(6) Siguiendo el ejemplo de la UFMG.

Scire. 12 : 2 (jul.-dic. 2006) 13-26. ISSN 1135-3716. 


\section{Referencias}

Abbot, A. (1988). The system of professions. Chicago: The University of Chicago Press, 1988.

Barbosa, M. L. O. (1993). A sociologia das profissões: em torno da legitimidade de um objeto. // BIB, 36 (2. sem. 1993) 3-30.

Boltanski, L. (1982). Les cadres, la formation d'un groupe social. París: Éditions de Minuit, 1982.

Bourdieu, P. (1989). O poder simbólico. Lisboa: Difel, 1989.

Brasil. Ministério do Trabalho e Emprego (2002). Classificação Brasileira de Ocupações. CBO-2002. http://www.mtecbo.gov.br/index.htm (2004-04-30).

CEDES - Centro de Estudos Educação e Sociedade (2004). Programa de pesquisa em ciência e tecnologia, qualificação e produção. http://www.cedes.unicamp.br/pesquisa.html (2004-04-30).

Chen, C.-C.; Raskin, S.; Tebbets, D. R. (1984). Products of graduate library and information science schools: unadapted resources? // Education for Information. 2:3 (Sept. 1984) 163-190.

Christensen, J. (1992). The information professional in Denmark. // FID. State of the modern information professional: 1992-1993. La Haya: FID, 1992. 67-92.

Crivellari, H. M. T. (2000). Profissão/ocupação. // Fidalgo, F.; Machado, L. (eds.). Dicionário da educação profissional. Belo Horizonte: NETE/FAE/UFMG, 2000.

Crivellari, H. M. T. (2003). Relação educativa e formação profissional na ciência da informação. // Anais do V ENANCIB - Encontro Nacional de Pesquisa em Ciência da Informação. Belo Horizonte: UFMG, 2003. 1 CD-ROM.

Cronin, B. (1988a). Post-professionalism. // Post-professionalism: transforming the information heartland. Londres: Taylor Graham, 1988. 277-285.

Cronin, B. (1988b). Nichemanship for the nineties. // Post-professionalism: transforming the information heartland. London: Taylor Graham, 1988. 328-332.

Cronin, B.; Stiffler, M.; Day, D. (1993). The emergent market for information professionals: educational opportunities and implications. // Library Trends. 42:2 (Fall 1993) 257-276.

Cunha, M. V. (2000). O profissional da informação: formação e mercado de trabalho: revisão de literatura. São Paulo: Associação Paulista de Bibliotecários, 2000.

Cunha, M. V. (2002). Le profil des professionnels de l'information dans l'État de Santa Catarina, Brésil. // Recherches récentes en sciences de l'information: convergences et dynamiques. Coloque MICS-LERASS (Toulouse, 2002). París: ADBS, 2002. 281-298.

Cunha, M. V. (2006). Espacios de trabajo para profesionales de la información en Brasil: resultados preliminares. // Scire: Representación y Organización del Conocimiento. 12:2 (jul.-dic. 2006). 27-36.

Cunha, M. V.; Pereira, M. C. (2003). O perfil do profissional da informação em Santa Catarina: primeiros resultados. // Anais do V ENANCIB - Encontro Nacional de Pesquisa em Ciência da Informação. Belo Horizonte: UFMG, 2003. 1 CD-ROM.

Curry, L.; Wergin, J. F. (1993). Setting priorities for change in professional education. // Educating professionals. San Francisco: Jossey-Bass, 1993. 316-327.

Scire. 12 : 2 (jul.-dic. 2006) 13-26. ISSN 1135-3716. 
Davies, J. E. (1990). Professional development and the Institute of Information Scientists. // Journal of Information Science, 16:6 (1990) 369-379.

Delorme, R.; André, C. (1983). L'État et l'économie. París: Seuil, 1983.

Freidson, E. (1988). Professional powers: a study of the institutionalization of formal knowledge. Chicago: The University of Chicago, 1988.

Haug, M. R. (1973). Deprofessionalization: an alternate hypothesis for the future. // The Society Review Monograph. 20 (Dec. 1973) 195-211.

Jannuzzi, P. M. (2003). Biblioteconomistas e outros profissionais da informação no mercado de trabalho brasileiro: 1980-1996. // Anais do V ENANCIB - Encontro Nacional de Pesquisa em Ciência da Informação. Belo Horizonte: UFMG, 2003. 1 CD-ROM.

Larson, M. S. (1977). The rise of professionalism. Princeton: University of California Press, 1977.

Moore, W. E. (1970). The professions: roles and rules. Nueva York: Russel Sage Foundation, 1970.

Rosemberg, D. S., et ál. (2003). O perfil do bibliotecário em exercício no Espírito Santo. // Anais do V ENANCIB - Encontro Nacional de Pesquisa em Ciência da Informação. Belo Horizonte: UFMG, 2003. 1 CD-ROM.

SENAI. Serviço Nacional de Aprendizagem Industrial (1998). Modernização, emprego e qualificação profissional. Río de Janeiro: SENAI/DN, 1998.

Sergean, R. (1977). Librarianship and information work: job characteristics and staffing needs. Boston Spa: The British Library Board, 1977. (The British Library Research and Development Reports, 5321HC).

Starr, K. (1996). Task force on the future of librarianship. Sacramento, CA: California Library Association, 1996.

Scire. 12 : 2 (jul.-dic. 2006) 13-26. ISSN 1135-3716. 